\title{
Exact ground state of the Lieb-Mattis Hamiltonian as a superposition of Néel states
}

\author{
Louk Rademaker \\ Department of Theoretical Physics, University of Geneva, 1211 Geneva, Switzerland
}

(Received 24 September 2019; revised manuscript received 28 October 2019; published 14 November 2019)

\begin{abstract}
We show that the exact ground state of the Lieb-Mattis Hamiltonian is an equal-weight superposition of all possible classical Néel states, and provide an exact formulation of this superposition in the $z$-spin basis for both $S=1 / 2$ and general $S$ using Schwinger bosons. In general, a superposition of possible rotations on a general initial state is symmetric if and only if the initial state has a nonzero overlap with a singlet state and is otherwise made up of states that vanish due to the symmetrization. Most notably, $|s, m=0\rangle$ states will vanish if symmetrized, which explains how a superposition of Néel states projects onto its singlet component.
\end{abstract}

DOI: 10.1103/PhysRevResearch.1.032018

\section{INTRODUCTION}

The ground state of finite systems that exhibit spontaneous symmetry breaking in the thermodynamic limit is typically still symmetric and unique. In particular, this has been proven for the Heisenberg antiferromagnet $[1,2]$. To arrive at such a proof of uniqueness, Lieb and Mattis considered only the $k=$ $0, \pi$ part of the Heisenberg Hamiltonian, which is now known as the Lieb-Mattis Hamiltonian. This model is defined for a spin system on a bipartite lattice, and reads

$$
\hat{H}_{\mathrm{LM}}=\frac{1}{N} \hat{\mathbf{S}}_{A} \cdot \hat{\mathbf{S}}_{B},
$$

where $\hat{\mathbf{S}}_{A / B}$ is the total spin on the $A / B$ sublattice, and $N$ is the total system size.

The Lieb-Mattis Hamiltonian provides an interesting exactly solvable toy model for studying the mechanism of spontaneous symmetry breaking [3]. Its spectrum forms the "tower of states" [4], a collection of low-lying excitations with a gap of the order $O(1 / N)$. Because this gap is so small, a tiny perturbation is sufficient to break the symmetry for sufficiently large systems. This singular behavior can be solved exactly for Eq. (1), but is generic among almost all forms of spontaneous symmetry breaking [5]. Explicitly, one can add a symmetry-breaking field $\hat{H}^{\prime}=-B\left(\hat{S}_{A}^{z}-\hat{S}_{B}^{z}\right)$ to the Lieb-Mattis model, and find that the limits $B \rightarrow 0^{+}$ and $N \rightarrow \infty$ do not commute. If one keeps $B$ finite while taking the thermodynamic limit, the resulting ground state is the classical Néel state antiferromagnet. A similar result is also found in more complicated models, such as the full Heisenberg model where the symmetry-breaking perturbation will force the system into a quantum antiferromagnetic state.

In the thermodynamic limit, therefore, a system that exhibits symmetry breaking has in general two types of ground

Published by the American Physical Society under the terms of the Creative Commons Attribution 4.0 International license. Further distribution of this work must maintain attribution to the author $(s)$ and the published article's title, journal citation, and DOI. states: the symmetric one, and the symmetry-broken one. What is the relation between these two? This question can be studied exactly in the context of the Lieb-Mattis toy model. In particular, can we take a suitable superposition of all possible Néel states to construct the symmetric Lieb-Mattis ground state? Here, we show that the answer to this question is yes. In fact, an equal-weight superposition of all possible directions of a Néel state is equal to the Lieb-Mattis ground state.

This led us to study more generally the properties of symmetric superpositions of some initial polarized state. We show that, depending on the definition of the rotation operator, some states vanish while others are projected onto states of the form $|s, m=0\rangle$. Consequently, we conclude that the "superposition of all directions" is symmetric if and only if the initial state has a nonzero overlap with a singlet and is otherwise made up of states that vanish.

In the remainder of this Rapid Communication we will first introduce rotations and the symmetrized superposition in Sec. II. In Sec. III we show, using three complementary approaches, that a superposition of Néel states is equal to the Lieb-Mattis ground state. In Sec. IV we derive the structure of a general superposition of directional states. Finally, in Sec. V we provide some outlook of our results.

\section{DEFINITIONS}

A state $|\psi\rangle$ is invariant under SU(2) rotations if and only if it is an eigenstate of every possible spin rotation. Note that due to the non-Abelian nature of SU(2), this implies that only states in the trivial representation, that is, singlet states with $\hat{\mathbf{S}}_{\text {tot }}^{2}=0$, are SU(2) invariant.

Consider a state $\left|\psi_{0}\right\rangle$ that is not $\mathrm{SU}(2)$ invariant. Without loss of generality, we consider it to have a polarization in the $z$ direction. Similar states in different directions can be constructed as follows,

$$
|\theta, \phi\rangle=e^{-i \phi \hat{S}^{z}} e^{-i \theta \hat{S}^{y}}\left|\psi_{0}\right\rangle
$$

which we will call a directional state. We define the symmetrized state as the equal-weight superposition of these 
different directional states,

$$
\left|\psi_{S}\right\rangle=\int \sin \theta d \theta d \phi|\theta, \phi\rangle .
$$

The definition of Eq. (2) is not unique. Though our definition is consistent with most of the literature (for example, Ref. [6]), in some instances a different definition of the spin rotation is chosen. For example, Ref. [7] defines directional states as

$$
|\theta, \phi\rangle_{G}=e^{\xi \hat{S}^{+}-\xi^{*} \hat{S}^{-}}|s, s\rangle,
$$

with $\xi=-\frac{\theta}{2} e^{-i \phi}$ and $|s, s\rangle$ the maximally polarized state with total spin $s$. These states differ a phase factor $e^{i s \phi}$ from our definition Eq. (2). However, as we shall see, for a superposition of Néel states these phase factors exactly cancel, and therefore Eqs. (4) and (2) yield the same results. The precise definition of rotation matters only for the more general superposition discussed in Sec. IV.

Furthermore, observe that if one chooses the original not $\mathrm{SU}(2)$ invariant state to be the maximally polarized state $|s, s\rangle$, the definition Eq. (2) defines spin coherent state. The symmetrized state of Eq. (3) is thus a superposition of all possible spin coherent states.

\section{SYMMETRIZED NÉEL WAVE FUNCTION}

In this section we will show that a symmetrized superposition over Néel states is equal to the ground state of the Lieb-Mattis Hamiltonian. For this, consider a spin $S$ system on a bipartite lattice. The Néel state polarized in the $z$ direction is a product state with $|S\rangle$ on all sites in sublattice $A$ and $|-S\rangle$ on all $B$ sites,

$$
\left|\psi_{N}\right\rangle=\prod_{i \in A}|S\rangle_{i} \prod_{j \in B}|-S\rangle_{j} .
$$

As mentioned in the Introduction, the Lieb-Mattis Hamiltonian is defined as

$$
\begin{aligned}
\hat{H}_{\mathrm{LM}} & =\frac{1}{N} \hat{\mathbf{S}}_{A} \cdot \hat{\mathbf{S}}_{B} \\
& =\frac{1}{2 N}\left(\hat{\mathbf{S}}_{\text {tot }}^{2}-\hat{\mathbf{S}}_{A}^{2}-\hat{\mathbf{S}}_{B}^{2}\right) .
\end{aligned}
$$

For a bipartite lattice with $N$ sites and on each site a spin $S$, the unique ground state is a total singlet state $\left(S_{\text {tot }}=0\right)$ with maximal sublattice spin $S_{A}=S_{B}=N S / 2$ and ground-state energy $E_{0}=-\frac{S}{2}\left(\frac{N S}{2}+1\right)$ [8]. In order to show that a state is the ground state, it suffices therefore to show that it has maximal sublattice spin and total spin zero.

\section{A. General structure}

The symmetrized superposition of Néel states in all possible directions is

$$
\left|\psi_{S}\right\rangle=\frac{1}{4 \pi} \int_{0}^{\pi} \sin \theta d \theta \int_{0}^{2 \pi} d \phi e^{-i \hat{S}_{\mathrm{tot}}^{z} \phi} e^{-i \hat{S}_{\mathrm{tot}}^{y} \theta}\left|\psi_{N}\right\rangle
$$

(see also Sec. 4.2 of Ref. [9]).

Our claim is that this state is the ground state of the LiebMattis Hamiltonian. Since $\hat{\mathbf{S}}_{A / B}$ commute with the rotation operator $e^{-i \hat{S}_{\mathrm{tot}}^{z} \phi} e^{-i \hat{S}_{\mathrm{tot}}^{y} \theta}$, and $\hat{\mathbf{S}}_{A / B}^{2}\left|\psi_{N}\right\rangle=(N S / 2)(N S / 2+1)$, it follows that $\left|\psi_{S}\right\rangle$ has maximal sublattice spin $S_{A / B}=N S / 2$.
The only remaining thing to prove is that $\left|\psi_{S}\right\rangle$ is nonzero and it has $S_{\text {tot }}=0$.

First, we use that fact that the Néel state can be obtained by projecting the Lieb-Mattis ground state $\left|\psi_{\mathrm{LM}}\right\rangle$ onto the space with $S_{A}^{z}=N S / 2$ and $S_{B}^{z}=-N S / 2$,

$$
\left|\psi_{N}\right\rangle=\hat{P}_{S_{A}^{z}=N S / 2} \hat{P}_{S_{B}^{z}=N S / 2}\left|\psi_{\mathrm{LM}}\right\rangle .
$$

It follows that $\left\langle\psi_{\mathrm{LM}} \mid \psi_{N}\right\rangle \neq 0$. The overlap between the symmetrized wave function $\left|\psi_{S}\right\rangle$ and the Lieb-Mattis ground state is therefore nonzero, too,

$$
\begin{aligned}
\left\langle\psi_{\mathrm{LM}} \mid \psi_{S}\right\rangle= & \frac{1}{4 \pi} \int_{0}^{\pi} \sin \theta d \theta \int_{0}^{2 \pi} d \phi \\
& \times\left\langle\psi_{\mathrm{LM}}\left|e^{-i \hat{S}_{\mathrm{tot}}^{z} \phi} e^{-i \hat{S}_{\mathrm{tot}}^{y} \theta}\right| \psi_{N}\right\rangle \\
= & \frac{1}{4 \pi} \int_{0}^{\pi} \sin \theta d \theta \int_{0}^{2 \pi} d \phi\left\langle\psi_{\mathrm{LM}} \mid \psi_{N}\right\rangle \\
= & \left\langle\psi_{\mathrm{LM}} \mid \psi_{N}\right\rangle \neq 0 .
\end{aligned}
$$

This implies that $\left|\psi_{S}\right\rangle$ itself is nonvanishing.

To prove that $\left|\psi_{S}\right\rangle$ is a total spin singlet, we observe that the projection $\hat{P}_{S_{A}^{z}=N S / 2} \hat{P}_{S_{B}^{z}=N S / 2}$ is a function that only depends on the $z$ component of the spin $\hat{S}_{\text {tot }}^{z}$. Let us call this function $W\left(\hat{S}_{1}^{z}, \ldots, \hat{S}_{N}^{z}\right)$. Let $\hat{U}$ be an arbitrary uniform spin rotation, such that $\hat{U} \hat{S}_{j}^{z} \hat{U}^{\dagger}=\mathbf{n} \cdot \hat{\mathbf{S}}_{j}$ for all $j$. Since the Lieb-Mattis ground state satisfies $\hat{U}\left|\psi_{\mathrm{LM}}\right\rangle=\left|\psi_{\mathrm{LM}}\right\rangle$, we have

$$
\begin{aligned}
& \hat{U} W\left(\hat{S}_{1}^{z}, \ldots, \hat{S}_{N}^{z}\right)\left|\psi_{\mathrm{LM}}\right\rangle \\
& \quad=\hat{U} W\left(\hat{S}_{1}^{z}, \ldots, \hat{S}_{N}^{z}\right) \hat{U}^{\dagger}\left|\psi_{\mathrm{LM}}\right\rangle \\
& \quad=W\left(\mathbf{n} \cdot \hat{\mathbf{S}}_{1}, \ldots, \mathbf{n} \cdot \hat{\mathbf{S}}_{N}\right)\left|\psi_{\mathrm{LM}}\right\rangle \equiv W_{\mathbf{n}}\left|\psi_{\mathrm{LM}}\right\rangle
\end{aligned}
$$

where $W_{\mathbf{n}}$ only depends on the direction $\mathbf{n}$ and not on the specific choice of rotation operator $\hat{U}$. It follows that specifically for the Néel state [10], the precise choice of rotation operator $\hat{U}$ is not relevant and we can express the symmetrized wave function as

$$
\left|\psi_{S}\right\rangle=\int_{|\mathbf{n}|=1} \frac{d \mathbf{n}}{4 \pi} W_{\mathbf{n}}\left|\psi_{\mathrm{LM}}\right\rangle,
$$

which is manifestly $\mathrm{SU}(2)$ invariant and therefore a singlet.

We have thus proven that $\left|\psi_{S}\right\rangle$ given by Eq. (8) is the ground state of the Lieb-Mattis Hamiltonian [11].

\section{B. Explicit construction for $S=1 / 2$}

The previous paragraph contained an elegant and general proof that the symmetrized state $\left|\psi_{S}\right\rangle$ is the ground state of the Lieb-Mattis Hamiltonian. Here, we will construct explicitly this symmetrized wave function for $S=1 / 2$. Since any Néel state is a product state, we can write down the rotated Néel state as

$$
|\theta, \phi\rangle=\prod_{i \in A}\left|\psi_{i A}(\theta, \phi)\right\rangle \prod_{j \in B}\left|\psi_{j B}(\theta, \phi)\right\rangle,
$$

where, for $S=1 / 2$,

$$
\left|\psi_{i A}(\theta, \phi)\right\rangle=e^{-i \phi / 2} \cos \frac{\theta}{2}\left|\uparrow_{i}\right\rangle+e^{i \phi / 2} \sin \frac{\theta}{2}\left|\downarrow_{i}\right\rangle
$$


and

$$
\left|\psi_{j B}(\theta, \phi)\right\rangle=-e^{-i \phi / 2} \sin \frac{\theta}{2}\left|\uparrow_{j}\right\rangle+e^{i \phi / 2} \cos \frac{\theta}{2}\left|\downarrow_{j}\right\rangle .
$$

Using this explicit expression, we can construct the symmetrized wave function $\left|\psi_{S}\right\rangle$ in the basis of spin configurations $\mathcal{C}$,

$$
\left|\psi_{S}\right\rangle=\sum_{\mathcal{C}} a_{\mathcal{C}}|\{\mathcal{C}\}\rangle
$$

in the given quantization axis (here chosen to be $z$ ). It follows directly from Eqs. (15)-(17) that the amplitude $a_{\mathcal{C}}$ only depends on the number of $\uparrow, \downarrow$ spins on the $A / B$ sublattices,

$$
\begin{aligned}
a_{\mathcal{C}}= & \int_{0}^{\pi} \sin \theta d \theta \int_{0}^{2 \pi} d \phi \\
& \times\left(e^{-i \phi / 2} \cos \frac{\theta}{2}\right)^{N_{A}^{\uparrow}}\left(e^{i \phi / 2} \sin \frac{\theta}{2}\right)^{N_{A}^{\downarrow}} \\
& \times\left(-e^{-i \phi / 2} \sin \frac{\theta}{2}\right)^{N_{B}^{\uparrow}}\left(e^{i \phi / 2} \cos \frac{\theta}{2}\right)^{N_{B}^{\downarrow}} .
\end{aligned}
$$

We will first do the integral over $\phi$. Because the number of sites $N$ is even, we notice that $-N_{A}^{\uparrow}+N_{A}^{\downarrow}-N_{B}^{\uparrow}+N_{B}^{\downarrow}=$ $N^{\uparrow}-N^{\downarrow}$ is even as well. This implies that the integral over $\phi$ vanishes unless $N^{\uparrow}=N^{\downarrow}$. This means that $\left|\psi_{S}\right\rangle$ is an eigenstate of $\hat{S}_{\text {tot }}^{z}$ with eigenvalue zero.

The $S_{\text {tot }}^{z}=0$ condition allows us to write all parameters $N_{A / B}^{\uparrow / \downarrow}$ strictly as a function of $N_{B}^{\uparrow}$ and $N$,

$$
\begin{gathered}
N_{A}^{\uparrow}=N / 2-N_{B}^{\uparrow}, \\
N_{A}^{\downarrow}=N_{B}^{\uparrow}, \\
N_{B}^{\downarrow}=N_{A}^{\uparrow}=N / 2-N_{B}^{\uparrow} .
\end{gathered}
$$

The remaining integral over $\theta$ is obtained by using the identity

$$
I_{\theta}=\int_{0}^{\pi} \sin \theta d \theta \cos ^{k} \frac{\theta}{2} \sin ^{m} \frac{\theta}{2}=\frac{2(k / 2) !(m / 2) !}{[(k+m) / 2+1] !} .
$$

Throwing out an overall $N_{B}^{\uparrow}$-independent prefactor, we find that the unnormalized amplitudes are

$$
a_{N_{B}^{\uparrow}} \propto \frac{(-1)^{N_{B}^{\uparrow}}}{\left(\begin{array}{c}
N / 2 \\
N_{B}^{\uparrow}
\end{array}\right)} .
$$

We only need to establish the normalization. To do so, observe that for a system with $N$ spins, the number of states with $S_{\text {tot }}^{z}=0$ and a given $N_{B}^{\uparrow}$ is $\left(\begin{array}{c}N / 2 \\ N_{B}^{\uparrow}\end{array}\right)^{2}$ [12]. This binomial precisely cancels the binomial in Eq. (24), and because there are $N / 2+$ 1 different possible sectors with fixed $N_{B}^{\uparrow}$, we conclude that the proper normalized amplitudes are

$$
a_{N_{B}^{\uparrow}}=\frac{(-1)^{N_{B}^{\uparrow}}}{\sqrt{N / 2+1}\left(\begin{array}{c}
N / 2 \\
N_{B}^{\uparrow}
\end{array}\right)} .
$$

Note that the sign of the amplitude is completely determined by $N_{B}^{\uparrow}$ according to Marshall's sign rule for the singlet ground state of antiferromagnetic systems [1].
Next, we will show that the symmetric wave function $\left|\psi_{S}\right\rangle$ from Eq. (18) with amplitudes Eq. (25) is the ground state of the Lieb-Mattis Hamiltonian. To do this, we explicitly write out Eq. (6),

$$
\hat{H}_{\mathrm{LM}}=\frac{1}{N} \sum_{i \in A, j \in B}\left[\hat{S}_{i}^{z} \hat{S}_{j}^{z}+\frac{1}{2}\left(\hat{S}_{i}^{+} \hat{S}_{j}^{-}+\hat{S}_{i}^{-} \hat{S}_{j}^{+}\right)\right] .
$$

We then compute the amplitudes $a_{\mathcal{C}}^{\prime}$ of each configuration $\mathcal{C}$ with given $N_{B}^{\uparrow}$ in the vector $\hat{H}_{\mathrm{LM}}\left|\psi_{S}\right\rangle=\sum_{\mathcal{C}} a_{\mathcal{C}}^{\prime}|\mathcal{C}\rangle$.

How $\hat{\mathbf{S}}_{i} \cdot \hat{\mathbf{S}}_{j}$ acts on a configuration $\mathcal{C}$ depends on the spins at the sites $i, j$. For any pair of sites $i \in A$ and $j \in B$, there is a probability $P_{\uparrow \uparrow}=\frac{2 N^{\uparrow}}{N}\left(1-\frac{2 N^{\uparrow}}{N}\right)$ that in this configuration $\mathcal{C}$ the state on $i, j$ is $\left|\uparrow_{i} \uparrow_{j}\right\rangle$. Similarly, we get $P_{\downarrow \downarrow}=P_{\uparrow \uparrow}, P_{\uparrow \downarrow}=$ $\left(1-\frac{2 N^{\uparrow}}{N}\right)^{2}$, and $P_{\downarrow \uparrow}=\left(\frac{2 N^{\uparrow}}{N}\right)^{2}$.

We can use these probabilities to compute the three contributions to $a_{\mathcal{C}}^{\prime}$. The diagonal part of $\hat{H}_{\mathrm{LM}}\left(\frac{1}{N} \sum_{i j} \hat{S}_{i}^{z} \hat{S}_{j}^{z}\right)$ yields, when acting on $\left|\psi_{S}\right\rangle$, the following contribution to $a_{\mathcal{C}}^{\prime}$,

$$
a_{N_{B}^{\uparrow}} \frac{1}{4 N}(N / 2)^{2}\left(P_{\uparrow \uparrow}+P_{\downarrow \downarrow}-P_{\downarrow \uparrow}-P_{\uparrow \downarrow}\right) .
$$

Here, the factor $\frac{1}{4 N}$ comes from acting with $\frac{1}{N} \hat{S}_{i}^{z} \hat{S}_{j}^{z}$, the $(N / 2)^{2}$ is the total number of pairs $i \in A, j \in B$, and $a_{N_{B}^{\uparrow}}$ is taken from Eq. (25).

The $\hat{S}_{i}^{-} \hat{S}_{j}^{+}$term takes a state from the $\left(N_{B}^{\uparrow}-1\right)$ sector and brings it into the $N_{B}^{\uparrow}$ sector. Therefore, our configuration $\mathcal{C}$ obtains a contribution

$$
a_{\left(N_{B}^{\uparrow}-1\right)} \frac{1}{2 N}(N / 2)^{2} P_{\downarrow \uparrow} .
$$

Similarly, the contribution from the $\left(N_{B}^{\uparrow}+1\right)$ sector equals

$$
a_{\left(N_{B}^{\uparrow}+1\right)} \frac{1}{2 N}(N / 2)^{2} P_{\uparrow \downarrow} .
$$

Summing these three contributions Eqs. (27)-(29), and using the identities

$$
\begin{gathered}
\frac{a_{\left(N_{B}^{\uparrow}-1\right)}}{a_{N_{B}^{\uparrow}}}=-\frac{N / 2-N_{B}^{\uparrow}+1}{N_{B}^{\uparrow}}, \\
\frac{a_{\left(N_{B}^{\uparrow}+1\right)}}{a_{N_{B}^{\uparrow}}}=-\frac{N_{B}^{\uparrow}+1}{N / 2-N_{B}^{\uparrow}},
\end{gathered}
$$

we find that the amplitude of the configuration $\mathcal{C}$ with given $N_{B}^{\uparrow}$ in the vector $\hat{H}_{\mathrm{LM}}\left|\psi_{S}\right\rangle$ equals

$$
a_{\mathcal{C}}^{\prime}=-\frac{1}{4}\left(\frac{N}{4}+1\right) a_{N_{B}^{\uparrow}}
$$

This proves that

$$
H_{\mathrm{LM}}\left|\psi_{S}\right\rangle=-\frac{1}{4}\left(\frac{N}{4}+1\right)\left|\psi_{S}\right\rangle,
$$

and thus that $\left|\psi_{S}\right\rangle$ is the ground state of the Lieb-Mattis Hamiltonian for $S=\frac{1}{2}$.

\section{Schwinger boson representation}

The construction for $S=1 / 2$ above can be extended to general $S$ using the method of Schwinger bosons [13]. In the 
Schwinger boson technique, one replaces the spin operators by two sets of bosons,

$$
\begin{gathered}
\hat{S}_{j}^{+}=\hat{a}_{j}^{\dagger} \hat{b}_{j}, \\
\hat{S}_{j}^{-}=\hat{b}_{j}^{\dagger} \hat{a}_{j}, \\
\hat{S}_{j}^{z}=\frac{1}{2}\left(\hat{a}_{j}^{\dagger} \hat{a}_{j}-\hat{b}_{j}^{\dagger} \hat{b}_{j}\right),
\end{gathered}
$$

under the constraint that $\hat{a}_{j}^{\dagger} \hat{a}_{j}+\hat{b}_{j}^{\dagger} \hat{b}_{j}=2 S$. A spin coherent state pointing in the $\mathbf{n}$ direction at site $j$ can be written using Schwinger bosons as

$$
|\mathbf{n}\rangle_{j}=\frac{\left(u \hat{a}_{j}^{\dagger}+v \hat{b}_{j}^{\dagger}\right)^{2 S}}{\sqrt{(2 S) !}}|0\rangle_{j}
$$

where $|0\rangle_{j}$ is the (unphysical) boson vacuum, $u=e^{i \phi / 2} \cos \frac{\theta}{2}$, and $v=e^{-i \phi / 2} \sin \frac{\theta}{2}$; compare to Eq. (16). A state in the opposite direction is expressed as

$$
|-\mathbf{n}\rangle_{j}=\frac{\left(i v^{*} \hat{a}_{j}^{\dagger}-i u^{*} \hat{b}_{j}^{\dagger}\right)^{2 S}}{\sqrt{(2 S) !}}|0\rangle_{j} .
$$

The Néel state for general $S$ can therefore be written as the product state of an $S_{A}=N S / 2$ spin coherent state on sublattice $A$ and an $S_{B}=N S / 2$ spin coherent on sublattice $B$ in the opposite direction,

$$
\left|\psi_{N}(\mathbf{n})\right\rangle=\frac{1}{(N S) !}\left(u \hat{a}_{A}^{\dagger}+v \hat{b}_{A}^{\dagger}\right)^{N S}\left(i v^{*} \hat{a}_{B}^{\dagger}-i u^{*} \hat{b}_{B}^{\dagger}\right)^{N S}|0\rangle,
$$

and the symmetrized state is now

$$
\left|\psi_{S}\right\rangle=\int_{|\mathbf{n}|=1} \frac{d \mathbf{n}}{4 \pi}\left|\psi_{N}(\mathbf{n})\right\rangle .
$$

Explicitly writing out this integral gives us

$$
\begin{aligned}
\left|\psi_{S}\right\rangle= & \frac{(-i)^{N S}}{4 \pi(N S) !} \sum_{k, \ell=0}^{N S}\left(\begin{array}{c}
N S \\
k
\end{array}\right)\left(\begin{array}{c}
N S \\
\ell
\end{array}\right) \\
& \times\left[\int_{0}^{2 \pi} d \phi \int_{0}^{\pi} d \theta \sin \theta u^{k} v^{N S-k}\left(-v^{*}\right)^{\ell}\left(u^{*}\right)^{N S-\ell}\right] \\
& \times\left(\hat{a}_{A}^{\dagger}\right)^{k}\left(\hat{b}_{A}^{\dagger}\right)^{N S-k}\left(\hat{a}_{B}^{\dagger}\right)^{\ell}\left(\hat{b}_{B}^{\dagger}\right)^{N S-\ell}|0\rangle .
\end{aligned}
$$

The part in the square brackets can only be nonzero when $k+$ $\ell-N S=0$ due to the integral over $\phi$. Eliminating $\ell$ yields an integral of the form (23),

$$
[\cdots]=2 \pi \int_{0}^{\pi} d \theta \sin \theta|u|^{2 k}|v|^{2(N S-k)}=\frac{2}{N S+1} \frac{1}{\left(\begin{array}{c}
N S \\
k
\end{array}\right)} .
$$

The full expression Eq. (41) now becomes

$$
\begin{aligned}
\left|\psi_{S}\right\rangle & =\frac{(-i)^{N S}}{(N S+1) !} \sum_{k=0}^{N S}\left(\begin{array}{c}
N S \\
k
\end{array}\right)(-1)^{N S-k}\left(\hat{a}_{A}^{\dagger} \hat{b}_{B}^{\dagger}\right)^{k}\left(\hat{b}_{A}^{\dagger} \hat{a}_{B}^{\dagger}\right)^{N S-k}|0\rangle \\
& =\frac{(-i)^{N S}}{(N S+1) !}\left(\hat{a}_{A}^{\dagger} \hat{b}_{B}^{\dagger}-\hat{b}_{A}^{\dagger} \hat{a}_{B}^{\dagger}\right)^{N S}|0\rangle .
\end{aligned}
$$

This final expression is the spin singlet ground state of the Lieb-Mattis Hamiltonian for general $S$ [14].

\section{GENERAL SYMMETRIZED SUPERPOSITIONS}

We showed that a symmetric superposition of Néel states yields the Lieb-Mattis Hamiltonian ground state. A natural follow-up question is, what happens if one takes a superposition of all possible directions of a general initial state?

To answer this question, we consider a spin state $\left|\psi_{0}\right\rangle$ that is somehow polarized in the $z$ direction. The classical Néel state polarized in the $z$ direction is an example of such a state, but one may also choose a ferromagnet in the $z$ direction, or any eigenstate of $S^{z}$ for a system of $N$ spin- $S$ degrees of freedom.

Now in general this initial state $\left|\psi_{0}\right\rangle$ is a superposition of states with different total spin $s$ and total magnetization $m$,

$$
\left|\psi_{0}\right\rangle=\sum_{s m} a_{s m}|s, m\rangle
$$

The total symmetrized state is thus a superposition of the symmetrized states expanded over this $s, m$ basis,

$$
\left|\psi_{S}\right\rangle=\sum_{s m} a_{s m}\left|\psi_{S}(s, m)\right\rangle,
$$

where we implicitly defined

$$
\left|\psi_{S}(s, m)\right\rangle=\int \sin \theta d \theta d \phi e^{-i \phi S^{z}} e^{-i \theta S^{y}}|s, m\rangle .
$$

There are four different ways $\left|\psi_{S}(s, m)\right\rangle$ can contribute to $\left|\psi_{S}\right\rangle$ :

(1) The symmetrized version of an initial singlet $|0,0\rangle$ is a singlet as well, so $\left|\psi_{S}(0,0)\right\rangle=|0,0\rangle$.

(2) The symmetrized version of an initial state $|s, m\rangle$ with $s>0$ and $m=0$ vanishes, $\left|\psi_{S}(s, m=0)\right\rangle=0$.

(3) The symmetrized version of an initial state $|s, m\rangle$ with $s>0$ and $m>0$ for $s-m$ even is proportional to the $m=0$ state, $\left|\psi_{S}(s, m)\right\rangle=|s, 0\rangle$.

(4) The symmetrized version of an initial state $|s, m\rangle$ with $s>0$ and $m>0$ for $s-m$ odd vanishes.

We can thus conclude that the final state $\left|\psi_{S}\right\rangle$ is symmetric if and only if the initial state has a nonzero overlap with a singlet and is otherwise made up of states that vanish.

For example, the Néel state is a superposition of the singlet state and other states with $s>0$ but $m=0$. Because $\mid s, m=$ $0)$ vanishes when averaged over, the final symmetrized state is just the singlet and hence symmetric. Notice that this is conform the notion of Anderson's tower of states, which expresses in general the symmetry broken state as a superposition of $m=0$ but $s \geqslant 0$ states [4].

A corollary of the above statement is that symmetrizing twice always projects the initial state onto its singlet component: The first symmetrization projects $\left|\psi_{0}\right\rangle$ onto its $m=0$ components, and the second symmetrization makes all terms vanish except for $s=0$. 
In order to prove the statements mentioned above, we will expand $e^{-i \phi S^{z}} e^{-i \theta S^{y}}|s, m\rangle$ in the basis of $\left|s, m^{\prime}\right\rangle$ states,

$$
\begin{aligned}
\left\langle s, m^{\prime}\left|e^{-i \phi S^{z}} e^{-i \theta S^{y}}\right| s, m\right\rangle & =e^{-i \phi m^{\prime}}\left\langle s, m^{\prime}\left|e^{-i \theta S^{y}}\right| s m\right\rangle \\
& =e^{-i \phi m^{\prime}} \sum_{x=\max \left(0, m^{\prime}-m\right)}^{\min \left(s-m, s+m^{\prime}\right)}(-1)^{x} \frac{\sqrt{(s+m) !(s-m) !\left(s+m^{\prime}\right) !\left(s-m^{\prime}\right) !}}{(s-m-x) !\left(s+m^{\prime}-x\right) ! x !\left(x+m-m^{\prime}\right) !} \cos ^{2 s+m^{\prime}-m-2 x} \frac{\theta}{2} \sin ^{2 x+m-m^{\prime}} \frac{\theta}{2} .
\end{aligned}
$$

The second line is based on Ref. [15], Eq. (15.27).

We can now write

$$
\left|\psi_{S}(s, m)\right\rangle=\sum_{m^{\prime}=-s}^{s}\left|s, m^{\prime}\right\rangle \int_{0}^{\pi} \sin \theta d \theta \int_{0}^{2 \pi} d \phi\left\langle s, m^{\prime}\left|e^{-i \phi S^{z}} e^{-i \theta S^{y}}\right| s, m\right\rangle .
$$

The integral over $\phi$ only yields a nonzero result whenever $m^{\prime}=0$ because of the phase factor $e^{-i \phi m^{\prime}}$. Note that here the explicit definition of the rotation comes into play: If we would use Eq. (4), the extra phase factor $e^{i s \phi}$ implies that we always project onto the $\left|s, m^{\prime}=s\right\rangle$ state. For our original definition of Eq. (2), we conclude that the symmetrized state is always a $m^{\prime}=0$ state,

$$
\left|\psi_{S}(s, m)\right\rangle=|s, 0\rangle \int_{0}^{\pi} \sin \theta d \theta\left\langle s, 0\left|e^{-i \theta S^{y}}\right| s, m\right\rangle .
$$

To see whether or not the prefactor vanishes, let us now compute the integral over $\theta$. For this, we use the identity of Eq. (23), so that, implicitly assuming $m \geqslant 0$,

$$
\begin{gathered}
\left|\psi_{S}(s, m)\right\rangle=|s, 0\rangle \sum_{x=0}^{s-m}(-1)^{x} \frac{s ! \sqrt{(s+m) !(s-m) !}}{(s-m-x) !(s-x) ! x !(x+m) !} I(2 s-m-2 x, 2 x+m) \\
=|s, 0\rangle \frac{2 \sqrt{(s+m) !(s-m) !}}{(s+1)} \sum_{x=0}^{s-m}(-1)^{x} \frac{(s-x-m / 2) !(x+m / 2) !}{(s-m-x) !(s-x) ! x !(x+m) !} .
\end{gathered}
$$

Now in a few limiting cases, this equation can be simplified dramatically. First, if our initial state was the $m=0$ state, we find that the sum over $x$ becomes $\sum_{x=0}^{s} \frac{(-1)^{x}}{(s-x) ! x !}=0$ for $s>0$. Therefore, the symmetrized state is actually vanishing.

The second limiting case is $m=s$, in which case

$$
\left|\psi_{S}(s, s)\right\rangle=|s, 0\rangle \frac{2 \sqrt{(2 s) !}}{(s+1)}\left(\frac{(s / 2) !}{s !}\right)^{2} .
$$

Third, observe that the sum over $x$ can be changed into a sum over $x^{\prime}=s-m-x$. This yields an extra factor of $(-1)^{s-m}$, but other than that, the expression is exactly the same in terms of $x$ or $x^{\prime}$. Therefore, if $s-m$ is an odd integer, the sum vanishes.

The final case, summarized by $0<m<s$ with $s-m$ an even integer, gives rise to a complicated expression,

$$
\left|\psi_{S}(s, m)\right\rangle=|s, 0\rangle \frac{2^{s-m} m(s / 2-1) !(s / 2) ![(s-m-1) / 2] ! \sqrt{(s+m) !}}{\sqrt{\pi}(s+1) s ![(s+m) / 2] ! \sqrt{(s-m) !}}
$$

which is clearly nonvanishing. This concludes the proof of the four statements at the beginning of this section.

\section{CONCLUSION AND OUTLOOK}

In this Rapid Communication we investigated the properties of a symmetric superposition of all possible directional states. In particular, we showed that a superposition of Néel states equals the exact symmetric ground state of the LiebMattis Hamiltonian.

Many models that exhibit spontaneous symmetry breaking have an exact symmetric ground state at any finite system size. Above this ground state is a spectrum of low-lying states whose gap scales as $O(1 / N)$ [5]. The symmetry-broken state can now be constructed as a superposition between the ground state and these low-lying states. Specifically for the Heisenberg model, it has been shown that the tower of states is described by the Lieb-Mattis Hamiltonian [2], whereas the higher excited states form the spectrum of spin waves. This distinction, between a $\Delta \sim O(1 / N)$ tower of states and Goldstone modes with a larger gap, is generic for spontaneous symmetry-breaking systems. For example, Ref. [5] discusses the tower of states for a Bose condensate, and in recent lecture notes this is worked out for the harmonic crystal [3]. With the advent of a more powerful numerical technique, one can even compute the low-lying spectrum exactly and from this infer the type of symmetry breaking [16].

It might seem counterintuitive that the infinite-range interacting Lieb-Mattis Hamiltonian describes properties of the short-range interacting Heisenberg model. However, 
the states in the tower of states all differ macroscopically from each other. Even though the full Heisenberg model is short ranged, an effective Hamiltonian for the tower of states is therefore long ranged. The major difference between the ground state of the Heisenberg model and that of the Lieb-Mattis model is the inclusion of finite-wave-number corrections, which can be captured in quite good accuracy using linear spin-wave theory [17].

We hypothesize that, in general, beyond just the exactly solvable Lieb-Mattis model for the tower of states, the symmetric ground state of a system exhibiting spontaneous symmetry breaking can be expressed as an equal-weight superposition of the symmetry-broken ground states. For example, linear spin-wave (LSW) theory provides us an approximation of a symmetry-broken ground state for the Heisenberg antiferromagnet [17]. Taking a superposition of LSW ground states in different directions will approximate the symmetric ground state of the Heisenberg Hamiltonian. We expect the same phenomenology for $X Y$ magnets or Bose condensates
[U(1) superfluids]. A possible extension of our work might include $\mathrm{SU}(n)$ symmetric systems.

A notable exception appears for so-called "type B" spontaneous symmetry breaking, such as ferromagnets $[18,19]$. Here, the order parameter commutes with the Hamiltonian so that the ground state, even for finite-size systems, is not unique. Consequently, there is no "symmetric" ground state and our results show that one cannot make a symmetric state by superposing, for example, ferromagnets in different directions. The same holds for other type B systems such as ferrimagnets [20].

\section{ACKNOWLEDGMENTS}

We thank Jasper van Wezel and Aron Beekman for collaboration on a related project that inspired this work. We also thank Hal Tasaki and Hosho Katsura for discussions. This work is supported by the Swiss National Science Foundation via an Ambizione grant (L.R.).
[1] W. Marshall, Proc. R. Soc. London, Ser. A 232, 48 (1955).

[2] E. H. Lieb and D. Mattis, J. Math. Phys. 3, 749 (1962).

[3] A. Beekman, L. Rademaker, and J. van Wezel, arXiv:1909.01820 [SciPost Phys. Lect. Notes (to be published)].

[4] P. W. Anderson, Phys. Rev. 86, 694 (1952).

[5] H. Tasaki, J. Stat. Phys. 174, 735 (2018).

[6] J. R. Klauder and B.-S. Kagerstam, Coherent States Application in Physics and Mathematical Physics (World Scientific, Singapore, 1985).

[7] J.-P. Gazeau, Coherent States in Quantum Physics (Wiley-VCH, Weinheim, 2009).

[8] J. van Wezel, Phys. Rev. B 78, 054301 (2008).

[9] H. Tasaki, Physics and mathematics of quantum many body systems (unpublished).

[10] And other states that can be expressed as $W\left(\hat{S}_{1}^{z}, \ldots, \hat{S}_{N}^{z}\right)\left|\psi_{0}\right\rangle$ with $W$ some function and $\left|\psi_{0}\right\rangle$ a singlet state.
[11] H. Tasaki (private communication).

[12] One can verify that indeed the total number of states with $S_{\text {tot }}^{z}=$ 0 is given by $\left(\begin{array}{c}N \\ N / 2\end{array}\right)=\sum_{N_{B}^{\uparrow}=0}^{N / 2}\left(\begin{array}{c}N / 2 \\ N_{B}^{\uparrow}\end{array}\right)^{2}$.

[13] A. Auerbach, Interacting Electrons and Quantum Magnetism (Springer, New York, 1994).

[14] H. Katsura (private communication).

[15] E. P. Wigner, Group Theory and its Application to the Quantum Mechanics of Atomic Spectra (Academic, London, 1959).

[16] A. Wietek, M. Schuler, and A. M. Läuchli, arXiv:1704.08622.

[17] E. Manousakis, Rev. Mod. Phys. 63, 1 (1991).

[18] H. Watanabe and H. Murayama, Phys. Rev. Lett. 108, 251602 (2012).

[19] H. Watanabe and H. Murayama, Phys. Rev. X 4, 031057 (2014).

[20] L. Rademaker, A. Beekman, and J. van Wezel, arXiv:1909.11381. 